\title{
InVESTIGATION OF ENDOBRONCHIAL TUBERCULOSIS Diagnoses in 22 CASES
}

\author{
X. Qingliang, W. Jianxin \\ The Respiratory Diseases Department of the Chinese PLA General Hospital, Beijing, China
}

\begin{abstract}
Background: Endobronchial tuberculosis (EBTB) is a special form of pulmonary tuberculosis. In spite of much progress in the diagnosis of this disease in past years, delayed or mistaken diagnosis is still commonly seen.

Objective: The aim of this study is to try to find out some useful clues for the diagnosis of EBTB, especially the early diagnosis.

Methods: The medical records of patients with EBTB were analyzed retrospectively. Results: The male-to-female ratio was $1: 2.2$ out of 22 patients. Patients aged below 60 -years-old constituted $72.7 \%$ of the cases. $22.7 \%$ of these patients were smokers. The male-to-female ratio of smokers was $4: 1.68 .2 \%$ of these patients tested all showed negative result for the HIV test. The frequent complaints were cough, sputum, shortness of breath and fever, and antibiotic treatments were usually inefficacious. Multiple lobes lesion, exudative shadow and atelectasis were the frequent radiological findings. Acid-fast bacilli staining for sputum smear was positive in only $13.6 \%$ of these patients. Tuberculin skin test was positive in $59.1 \%$ of these patients. Granular lesion was the most common bronchoscopic appearance in these patients. Histological changes showed distinctive tuberculose lesion in $72.2 \%$ of 18 patients undergoing bronchoscopic biopsy.

Conclusion: The diagnosis of EBTB is easily delayed or mistaken because of nonspecific clinical manifestations and the low incidence of positive acid-fast bacilli staining. A high index of awareness of this disease is required for diagnosis. Bronchoscopy should be performed as soon as possible in suspected patients, especially when patients present positive tuberculin skin test or no response to antibiotic treatments.
\end{abstract}

Key words: endobronchial tuberculosis; diagnosis

\section{INTRODUCTION}

Pulmonary tuberculosis is one of the major health problems worldwide [1]. In spite of much progress in diagnosis and therapy, this problem still remains. Moreover, there has been a resurgence of pulmonary tuberculosis recently, which is related to the HIV epidemic, multidrug-resistant strains, poverty, immigration, and shortness in the prevention and treatment system [2-4]. Endobronchial tuberculosis (EBTB) is a special form of pulmonary tuberculosis. About $10 \%$ to $40 \%$ of patients with active pulmonary tuberculosis had EBTB as reported in previous studies $[5,6]$. The diagnosis of typical pulmonary tuberculosis is easily confirmed by bacteriological means and radiological findings. However, the diagnosis of EBTB is more difficult because of variable clinical manifestations.

One reason of EBTB assuming importance is the diagnosis is easily delayed and mistaken. The other is EBTB may cause a long term sequelae of bronchostenosis. The bronchostenosis is mainly determined by the extent of disease progression and closely related to the formation of granulation tissue $[7,8]$. Once fibrostenosis already develops or extensive granulation tissue appears, remarkable bronchostenosis is inevitable despite of efficacious anti-tuberculosis chemotherapy [9-11]. The incidence rate of bronchostenosis may reach up to $68 \%$ in initial 4 to 6 months of the disease and rises further with the course of disease elongating [12]. Severe bronchostenosis may cause pulmonary complications such as pulmonary infection, atelectasis, bronchiectasis, and even death by inducing respiratory failure and asphyxia. Additionally, the bronchostenosis may cause intractable tuberculosis and make patients become chronic infection sources of tuberculosis.

The eradication of tubercle bacilli and the prevention of bronchostenosis are the two most substantial goals of EBTB treatment. To get treatment goals, the diagnosis of this disease must be established early and aggressive treatments must be performed before the disease progresses too far. In this study, by analyzing the cases of EBTB, we tried to find out some useful clues for the diagnosis of this disease, especially the early diagnosis.

\section{Patients and Methods}

Out of 1,222 patients diagnosed with pulmonary tuberculosis from 1995 to 2008 in the Chinese PLA General Hospital, a tertiary care center, 22 patients were diagnosed as EBTB. The diagnosis of EBTB was established with the combination of: positive acid-fast bacilli in sputum smear; histological tuberculous lesion confirmed by biopsy after bronchoscopy or pulmonary lobectomy; typical bronchial mucosal changes seen on bronchoscopy; clinical and radiological findings; treatment outcome.

All of these patients underwent the examinations of acid-fast bacilli in sputum smear, tuberculin skin test, 
chest radioscopy and bronchoscopy, 18 patients underwent the bronchoscopic biopsy, and 4 patients underwent the biopsy after pulmonary lobectomy. We reviewed these patient's medical records, radiological findings, bronchoscopic features and pathological changes, and retrospectivly analyzed these cases.

\section{RESULTS}

4 patients were male, 18 patients were female and the male-to-female ratio was 1:2.2 out of 22 patients. Their mean age was 46.8 years with a range from 23 to 82 years. 16 patients $(72.7 \%)$ were younger than 60 years-old, and 6 patients $(27.3 \%)$ were older than 60 years-old. 5 patients $(22.7 \%)$ were smokers, including all of the 4 male patients and 1 female patient. The male-to-female ratio of smokers was $4: 1$. Their mean smoking history was 17-pack-year with a range from 10 to 30 -pack-year. 15 patients $(68.2 \%)$ undergoing the serologic examination of HIV antibody all tested negative.

The chief complaints were cough $(95.5 \%)$, sputum $(63.6 \%)$, shortness of breath $(36.4 \%)$, fever $(27.3 \%)$, hemoptysis $(18.2 \%)$ and wheezing $(13.6 \%)$. One patient was asymptomatic. The frequent signs were diminished breath sound $(31.8 \%)$, respiratory rudeness $(13.6 \%)$ and rhonchus (9.1\%). 10 patients $(45.5 \%)$ had negative signs. 3 patients $(13.6 \%)$ had past history of tuberculosis. Most of these patients ever received antibiotic treatments, but were inefficacious. The duration of symptoms before visiting our hospital ranged from 7 days to 3 years, and the duration of 10 patients (45.5\%) were longer than 3 months.

Radiological findings showed varied pulmonary lesions (Fig. 1) in all of these patients except one who had normal chest radiograph. The pulmonary lesions occurred at the distinctive site of tuberculosis only in 4 patients $(18.2 \%)$, and non-distinctive sites in $17 \mathrm{pa}$ tients $(77.3 \%)$. Multiple lobes were involved in $12 \mathrm{pa}$ tients $(54.5 \%)$, left lower lobe in 2 patients $(9.1 \%)$, left lingual lobe in 1 patient $(4.5 \%)$, right lower lobe in 1 patient $(4.5 \%)$ and right middle lobe in 1 patient $(4.5 \%)$. Larger-area exudative shadow $(36.4 \%)$, atelectasis $(27.3 \%)$ and multiple nodules $(18.2 \%)$ were the common radiological findings. The thickening of the bronchial wall and pleural effusion could be seen in 3 patients $(13.6 \%)$ and 2 patients $(9.1 \%)$ respectively. Different radiological features could be often seen in the same patient.

Acid-fast bacilli staining for sputum smear was positive in only 3 patients $(13.6 \%)$. However, tuberculin skin tests were positive in 13 patients $(59.1 \%)$ and the indurations of 11 patients $(50.0 \%)$ were larger than $5 \mathrm{~mm}$ in diameter.

The forms of EBTB could be classified into seven subtypes by bronchoscopic features according to the previous studies $[12,13]$. If multiple bronchial mucosal lesions occurred in the same patient, the most obvious lesion was the basis of classification. In our study, the granular type $(31.8 \%)$ was the most common subtype, and followed by the nonspecific bronchitic $(18.2 \%)$, the tumorous $(13.6 \%)$, the fibrostenotic $(13.6 \%)$, the edematous-hyperemic $(9.1 \%)$, the actively caseating $(9.1 \%)$ and the ulcerative $(4.5 \%)$.

Histological changes showed distinctive tuberculose lesion in $72.2 \%$ of 18 patients undergoing bronchoscopic biopsy, and in all of the remaining 4 patients undergoing biopsy after pulmonary lobectomy.

\section{DisCusSION}

EBTB is defined as the tuberculous infection of tracheobronchial tree. It can affect any part and layer of the tracheobronchial wall. The incidence of EBTB is increasing in recent years, especially with the impact of HIV infection [3, 14]. The pathogenesis of EBTB is not fully clear. Possible mechanisms include direct implantation of tubercle bacilli from parenchymal focus, direct infiltration from adjacent tuberculous mediastinal lymph node, rupture of tuberculous intrathoracic lymph node into the bronchus, lymphatic spread along the bronchial tree by lymphatic drainage, and

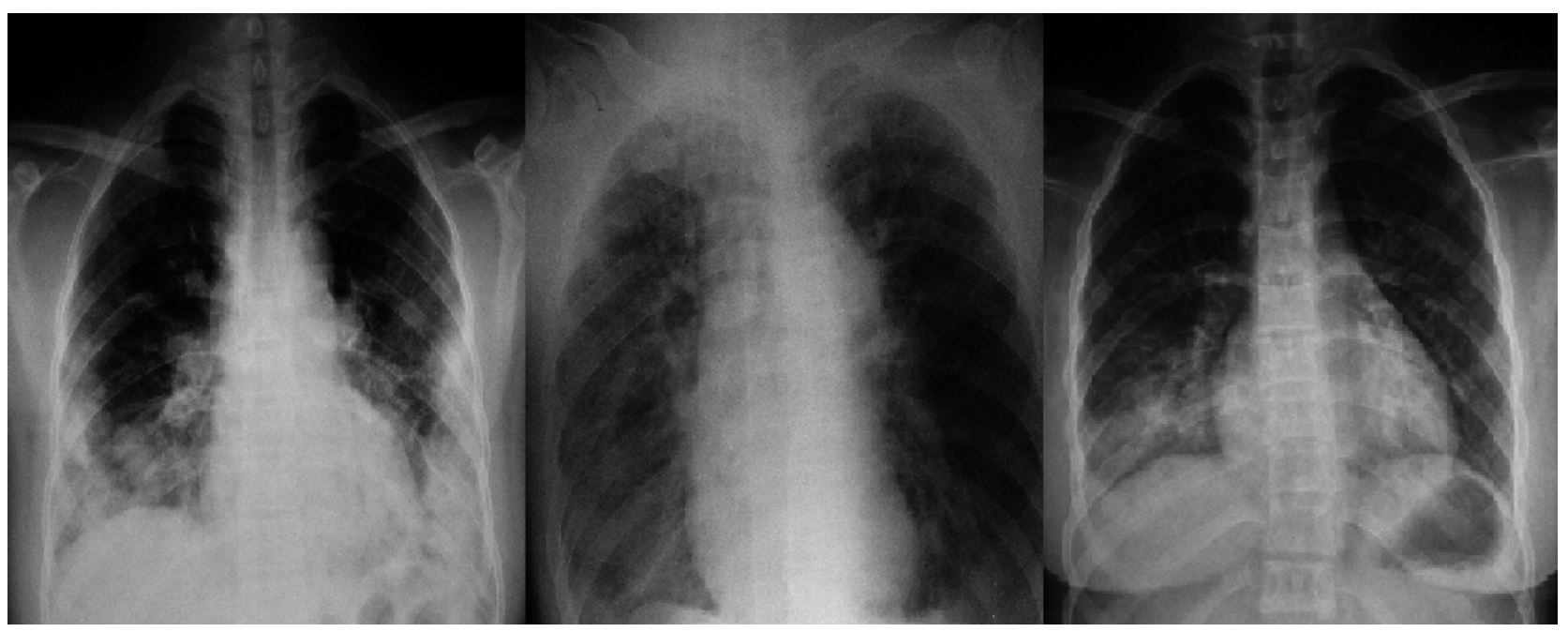

Fig 1. Radiological findings showed varied pulmonary lesions in patients with EBTB. The left: sheeted exudative shadows and nodules in bilateral middle and lower lung fields. The middle: atelectasis of right lung. The right: exudative shadows in right lower lung field. 
hematogenous spread. Most EBTB cases have obvious pulmonary lesions, but $10 \%$ to $20 \%$ of the cases may have normal chest radiographs [15]. EBTB usually complicates the pulmonary lesions and simulates other diseases [15-18], and other diseases may also simulate EBTB [19], all of which can lead to delayed or mistaken diagnosis. Early diagnosis is more difficult in such patients with normal chest radiographs [20]. Delayed or mistaken diagnosis and treatments rise to the incidence of bronchostenosis which is relate to the degree, extent and duration of lesions before treatment [13]. To avoid delayed diagnosis and treatments, it is required to study the clinical manifestations of patients with EBTB.

In this study, only 22 patients were diagnosed as EBTB out of 1,222 patients with pulmonary tuberculosis. This was a very low ratio compared with the incidence rate reported previously $[5,6]$, which suggested EBTB was often overlooked by clinicians. Thus, a high index of awareness of this disease is required for early diagnosis, especially in patients with negative HIV test because $68.2 \%$ of these patients undergoing HIV test all tested negative in this study.

Usually, EBTB is more commonly observed in female patients, of which the reasons are unclear. The possible reasons may include that females do not expectorate sputum as well as males because of their thinner bronchcial lumens and the sociocultural and cosmetic backgrounds. In this study, EBTB showed likewise preponderance in female patients, consistenting with other studies $[12,13]$. It is well-known that the incidence of EBTB is high in young patients. However, recent reports showed it was also high in elderly patients [21]. In this study, there were also 6 patients $(27.3 \%)$ who were older than 60 -years-old. The possible reasons of high EBTB incidence in elderly patients may include the diminished immune function causing reactivation of dormant tubercle bacilli and reinfection by new exogenous tubercle bacilli. Recent reports suggested that exogenous reinfection was a significant cause of tuberculosis in adults [22-25]. The high incidence of EBTB in female and elderly patients should be paid attention to.

It is well-known that smoking increases the risk for tuberculosis infection. However, it is unclear if smoking increases the incidence rate of EBTB. In this study, 5 patients $(22.7 \%)$ including all of the 4 male patients and 1 female patient were smokers and the male-to-female ratio of smokers was $4: 1$. This suggested that smoking might be a predominant high risk factor for EBTB in males, but not in females.

The clinical manifestations of EBTB may be acute, insidious, or delayed [26]. In this study, the frequent complaints and signs were varied and no helpful to distinguish EBTB from other diseases, let alone such patients without symptoms and positive signs. In this study, only $18.2 \%$ of these patients presented hemoptysis, a frequent symptom of pulmonary tuberculosis. This suggested hemoptysis was also not helpful to the diagnosis of EBTB. However, the inefficacy of antibiotic treatments and the relatively long duration of symptoms might be clues for diagnosis of EBTB.

Most cases of EBTB have concurrent pulmonary lesions and the radiological findings are usually mis-

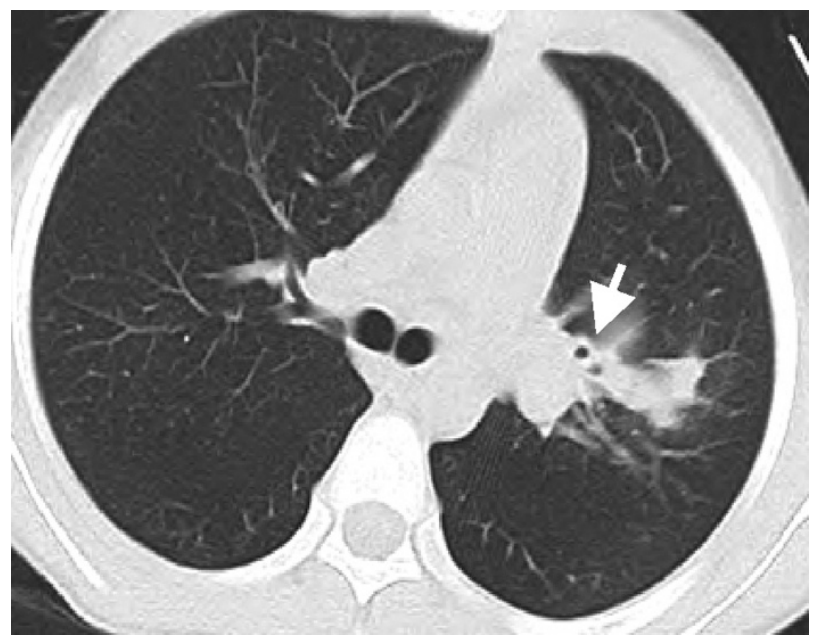

Fig. 2. Computer tomography of a patient with EBTB showed the wall thickening of left upper bronchi (arrow) and exudative shadow in left upper lobe.

leading. In our study, all of these patients presented varied pulmonary lesions except one with normal chest radiograph. Most pulmonary lesions of these patients located the non-distinctive site and showed no characteristics of tuberculosis. Multiple lobes lesion, exudative shadow and atelectasis were more commonly seen. These radiological findings could make it difficult to diagnose early. Computerized tomography may find the lesions of bronchus (Fig. 2) and be helpful to the diagnosis of EBTB [27, 28].

One of the important diagnosis means of EBTB is bacteriologic confirmation. Freshly expectorated sputum of patients is the best sample to stain for tubercle bacilli. However, the incidence of positive acid-fast bacilli staining in sputum smear is low. In our study, Only 3 patients $(13.6 \%)$ had positive acid-fast bacilli staining in sputum smear. An interesting finding in our study was that more than half of these patients $(59.1 \%)$ had positive tuberculin skin tests. Positive tuberculin skin tests were not attributed to the vaccination of Bacille Calmette-Guerin after five years duration since the last vaccination because the effects of vaccination generally wane with time [29] and adults seldom received the vaccination. Thus, we consider that the positive tuberculin skin test is a valuable clue for early diagnosis of EBTB.

The bronchoscopic appearances (Fig. 3) of EBTB could be classified into seven subtypes $[12,13]$ : the actively caseating (Bronchial mucosa was swollen, hyperemic and covered with a larger amount of whitish cheese-like material.), the oedematous-hyperaemic (Bronchial mucosal was severely swollen and hyperemic.), the fibrostenotic (Bronchial lumen narrowed obviously due to the fibrous hyperplasia and contracture.), the tumorous (Hyperplastic focal tissue formed an endobronchial mass like lung cancer.), the granular (Bronchial mucosa appeared severe inflammatory changes and rice-like nodules scattered in mucosa.), the ulcerative (Bronchial mucosa appeared ulcer.) and the nonspecific bronchitic (Bronchial mucosa only showed mild mucosal swelling and/or hyperemia). The subtype of the actively caseating is the most commonly seen as 


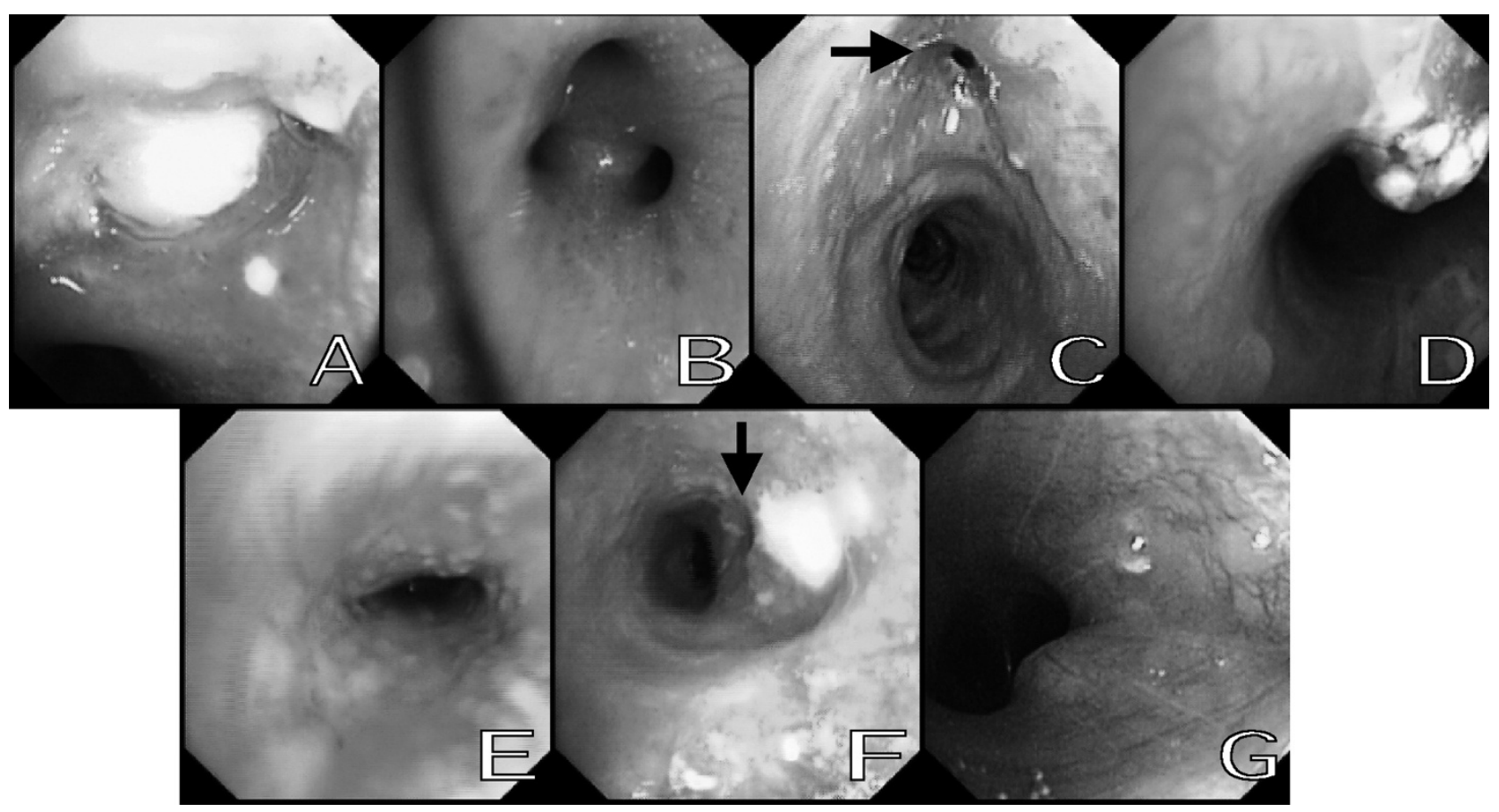

Fig. 3. Bronchoscopic appearances of seven subtypes of EBTB. A: the actively caseating. B: The oedematous-hyperaemic. C: The fibrostenotic (arrow). D: The tumorous. E: The granular. F: The ulcerative (arrow). G: The nonspecific bronchitic.

previously reported. However, in this study, the subtype of the granular $(31.8 \%)$ was most commonly seen, which differed from other studies. One of the possible reasons of this difference may be different pathogenesis and subpopulation. In this study, the diagnoses of EBTB in $72.2 \%$ of 18 patients undergoing bronchoscopic biopsy were confirmed by histological examination. This revealed bronchoscopic biopsy was a very valuable method for the diagnosis of EBTB, even in the patients with negative acid-fast bacilli staining in sputum smear. Other bronchoscopic procedures such as bronchoalveolar lavage, brushings, needle aspiration and endobronchial ultrasonography can also be used to increase the diagnostic success [30-35].

In conclusion, the diagnosis of EBTB is easily delayed or mistaken because of nonspecific clinical manifestations, misleading radiological findings and the low incidence of positive acid-fast bacilli staining. A high index of awareness is required for the diagnosis of this disease, especially the early diagnosis. The symptoms of refractory cough, sputum, shortness of breath, hemoptysis or wheezing, the signs of diminished breath sound, respiratory rudeness or rhonchus and the radiological findings of multiple lobes lesion, exudative shadow or atelectasis should be suspected caused potentially by EBTB. Bronchoscopy should be performed as soon as possible in suspected patients, especially when patients present the relatively long duration of symptoms, positive tuberculin skin test or no response to antibiotic treatments. If the diagnosis is not confirmed by pathological and bacteriological findings after repetitious biopsies and bacteriologic examinations, the diagnosis of EBTB should be established by the combination of clinical, radiological and bronchoscopic findings for early empirical treatments to avoid fibrous bronchostenosis.

\section{REFERENCES}

1. Dye C, Scheele S, Dolin P, et al. Global burden of tuberculosis: Estimated incidence, prevalence, and mortality by country. WHO Global Surveillance and Monitoring Project. JAMA; 1999, 282: 677-686.

2. Millard PS, Cegielski JP, Wing S, et al. Rurality and tuberculosis incidence trends in North and South Carolina, 1982 to 1992. J Rural Health; 1994, 10: 226-236.

3. Glynn JR. Resurgence of tuberculosis and the impact of HIV infection. Br Med Bull; 1998, 54: 579-593.

4. Lerner BH. Catching patients: tuberculosis and detention in the 1990s. Chest; 1999, 115: 236-241.

5. Calpe JL, Chiner E, Larramendi CH. Endobronchial tuberculosis in HIV-infected patients. AIDS; 1995, 9: 59-64.

6. Han JK, Im JG, Park JH, et al. Bronchial stenosis due to endobronchial tuberculosis: successful treatment with self-expandingmetallic stent. AJR; 1992, 159: 971-972.

7. Kim YH, Kim HT, Lee KS, et al. Serial fiberoptic bronchoscopic observations of endobronchial tuberculosis befor and early after antituberculosis chemotherapy. Chest; 1993, 103: 673-677.

8. Smith LS, Schillaci RF, Sarlin RF. Endobronchial tuberculosis: serial fiberoptic bronchoscopy and natural history. Chest; 1987, 91: 644-647.

9. Park CS, Him KU, Lee SM, et al. Bronchial hyperreactivity in patients with endobronchial tuberculosis. Respir Med; 1995, 89: 419-422.

10. Hoheisel G, Chan BK, Chan CH, et al. Endobronchial tuberculosis: diagnostic features and therapeutic outcome. Respir Med; 1994, 88: 593-597.

11. Park IW, Choi BW, Hue SH. Prospective study of corticosteroid as an adjunct in the treatment of endobronchial tuberculosis in adults. Respirology; 1997, 2: 275-281.

12. Lee JH, Park SS, Lee DH, et al. Endobronchial tuberculosis: clinical and bronchoscopic feature in 121 cases. Chest; 1992, 102: 990_993.

13. Chung HS, Lee JH. Bronchoscopic Assessment of the Evolution of Endobronchial Tuberculosis. Chest; 2000, 117: 385-392. 
14. Judson, MA, Sahn, SA. Endobronchial lesion in HIV-infected individuals. Chest; 1994, 105: 1314-1323.

15. YH Lee, KN Sin Fai Lam. Endobronchial tuberculosis simulating bronchial asthma. Singapore Med J; 2004, 45: 390-392.

16. Singla R, Kumar A, Chauhan D, et al. Endobronchial tuberculosis presenting as tumorous mass. Indian $\mathrm{J}$ Chest Dis Allied Sci; 2007, 49: 45-47.

17. Yuan YR, Liang BM, Zhu H, et al. Bronchial hyperreactivity in endobronchial tuberculosis. Zhonghua Jie He $\mathrm{He}$ Hu Xi Za Zhi; 2006, 29: 600-602.

18. Kreisel D, Arora N, Weisenberg SA, et al. Tuberculosis presenting as an endobronchial mass. J Thorac Cardiovasc Surg; 2007, 133: 582-584.

19. Rad MH, Milani M. Primary endobronchial actinomycosis simulating endobronchial tuberculosis in a patient with diabetes mellitus. Tuberk Toraks; 2007, 55: 186-190.

20. Taniguchi H, Suzuki K, Fujisaka S, et al. X-ray-negative endobronchial tuberculosis with persistent irritating cough that resulted in unpredicted mass infection. Nihon Kokyuki Gakkai Zasshi; 2003, 41: 541-545.

21. Kim HC, Kim HS, Lee SJ, et al. Endobronchial Tuberculosis Presenting as Right Middle Lobe Syndrome: Clinical Characteristics and Bronchoscopic Findings in 22 Cases. Yonsei Med J; 2008, 49: 615-619.

22. Chiang CY, Riley LW. Exogenous reinfection in tuberculosis. Lancet Infect Dis; 2005, 5: 629-636.

23. Verver S, Warren RM, Beyers N, Richardson M, et al. Rate of reinfection tuberculosis after successful treatment is higher than rate of new tuberculosis. Am J Respir Crit Care Med; 2005, 171: 1430-1435.

24. Van Rie A, Warren R, Richardson M, et al. Exogenous reinfection as a cause of recurrent tuberculosis after curative treatment. N Engl J Med; 1999, 341: 1174-1179.

25. Du Plessis DG, Warren R, Richardson M, et al. Demonstration of reinfection and reactivation in HIV-negative autopsied cases of secondary tuberculosis: multilesional genotyping of Mycobacterium tuberculosis utilizing IS6110 and other repetitive element-based DNA fingerprinting. Tuberculosis; 2001, 81: 211-220.

26. Caun HS, Sun A, Hoheisel GB. Endobronchial tuberculosis-is corticosteroid treatment useful?-a report of 8 cases and review of the literature. Postgrad Med J; 1990, 66: 822-826.
27. Park HJ, Park SH, Im SA, et al. CT differentiation of anthracofibrosis from endobronchial tuberculosis. AJR Am J Roentgenol; 2008, 191: 247-251.

28. Tong SM, Liu H. Spiral CT evaluation in diagnosis of endobronchial tuberculosis. Hunan Yi Ke Da Xue Xue Bao; 2001, 26: 165-166.

29. _er A, Metin A, Leyla S, et al. The diagnostic value of bronchoscopy in smear negative cases with pulmonary tuberculosis. Tüberküloz ve Toraks Dergisi; 2008, 56: 150157.

30. Saglam L, Akgun M, Aktas E. Induced sputum and bronchoscopy specimens in the diagnosis of tuberculosis. J Int Med Res; 2005, 33: 260-265.

31. Fujii H, Ishihara J, Fukaura A, et al. Early diagnosis of tuberculosis by fiberoptic bronchoscopy. Tuber Lung Dis; 1992, 73: 167-169.

32. Chawla R, Pant K, Jaggi OP, et al. Fibreoptic bronchoscopy in smear-negative pulmonary tuberculosis. Eur Respir J; 1998, 1: 804-806.

33. Charoenratanakul S, Dejsomritrutai W, Chaiprasert A. Diagnostic role of fiberoptic bronchoscopy in suspected smear negative pulmonary tuberculosis. Respir Med; 1995, 89: 621-623.

34. Tueller C, Chhajed PN, Buitrago-Tellez C, et al. Value of smear and PCR in bronchoalveolar lavage fluid in culture positive pulmonary tuberculosis. Eur Respir J; 2005, 26: 767-772.

35. Lin SM, Chung FT, Huang CD, et al. Diagnostic value of endobronchial ultrasonography for pulmonary tuberculosis. J Thorac Cardiovasc Surg; 2009, 138: 179-184.

Received: October 30, 2009 / Accepted: January 27, 2010

Address for correspondece:

Wang Jianxin

28 Fuxing Rd

Post code 100853

Beijing

China

Fax: 86-10-66939418

E-mail: wjx301@yahoo.com.cn; XQL0121@sohu.com 